\title{
The transiting planet OGLE-TR-132b revisited with new spectroscopy and deconvolution photometry ${ }^{\star} \star \star \star$
}

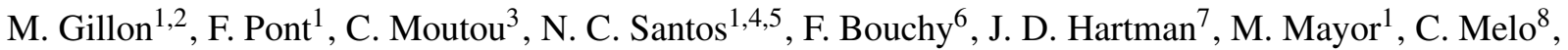 \\ D. Queloz ${ }^{1}$, S. Udry ${ }^{1}$, and P. Magain ${ }^{2}$
}

1 Observatoire de Genève, 51 Chemin des Maillettes, 1290 Sauverny, Switzerland e-mail: michael.gillon@obs.unige.ch

2 Institut d'Astrophysique et de Géophysique, Université de Liège, Allée du 6 Août 17, 4000 Liège, Belgium

3 LAM, Traverse du Siphon, BP8, Les Trois Lucs, 13376 Marseille Cedex 12, France

4 Centro de Astronomia e Astrofísica da Universidade de Lisboa, Observatório Astronómico de Lisboa, Tapada da Ajuda, 1349-018 Lisboa, Portugal

5 Centro de Geofisica de Évora, Rua Romão Ramalho 59, 7002-554 Évora, Portugal

6 Institut d'Astrophysique de Paris, UMR7095 CNRS, Université Pierre \& Marie Curie, 98bis Bd Arago, 75014 Paris, France

7 Harvard-Smithsonian Center for Astrophysics, 60 Garden Street, Cambridge, MA 02138, USA

8 European Southern Observatory, Casilla 19001, Santiago 19, Chile

Received 8 September 2006 / Accepted 5 February 2007

\begin{abstract}
OGLE-TR-132b transits a very metal-rich F dwarf about 2000 pc from the Sun, in the Galactic disc towards Carina. It orbits very close to its host $\operatorname{star}(a=0.03 \mathrm{AU})$ and has an equilibrium temperature of nearly $2000 \mathrm{~K}$. Using rapid-cadence transit photometry from the FORS2 camera on the VLT and SUSI2 on the NTT, and high-resolution spectroscopy with UVES on the VLT, we refine the shape of the transit light curve and the parameters of the system. In particular, we improve the planetary radius estimate, $R=1.18 \pm 0.07 R_{\mathrm{J}}$ and provide very precise ephemeris, $T_{\mathrm{tr}}=2453142.59123 \pm 0.0003 \mathrm{BJD}$ and $P=1.689868 \pm 0.000003$ days. The obtained planetary mass is $1.14 \pm 0.12 M_{\mathrm{J}}$. Our results give a slightly smaller and lighter star, and bigger planet, than previous values. As the VLT/FORS2 light curve obtained in this analysis with the deconvolution photometry algorithm DECPHOT shows a transit depth in disagreement with the one obtained by a previous study using the same data, we analyze them with two other reduction methods (aperture and image subtraction). The light curves obtained with the three methods are in good agreement, though deconvolution-based photometry is significantly more precise. It appears from these results that the smaller transit depth obtained in the previous study was due to a normalisation problem inherent to the reduction procedure used.
\end{abstract}

Key words. planetary systems - stars: individual: OGLE-TR-132 - techniques: photometric - techniques: image processing methods: data analysis

\section{Introduction}

Fourteen transiting extrasolar planets are now known, and they have proved invaluable in the study of hot Jupiter structure and evolution (Moutou \& Pont 2006). OGLE-TR-132b is a very short-period hot Jupiter $(P=1.69$ days $)$ orbiting a very metalrich $\mathrm{F}$ dwarf $([\mathrm{Fe} / \mathrm{H}]=0.37)$. The photometric transit signal was detected by Udalski et al. (2003) from the OGLE planetary transit search on the Warsaw $1.3 \mathrm{~m}$ telescope at Las Campanas, Chile. The spectroscopic orbit was measured by Bouchy et al. (2004), establishing the planetary mass of the transiting companion. High-precision photometry of the transit was reported by

* Based on data collected with the FORS2 imager at the VLTUT4 telescope (Paranal Observatory, ESO, Chile) in the programme 273.C-5017A, with the SUSI2 imager at the NTT telescope (La Silla Observatory, ESO, Chile) in the programme 075.C-0462A, and with the UVES spectrograph at the VLT-UT2 telescope (Paranal Observatory, ESO, Chile) in the programme 076.C-0131.

$\star \star$ The reduced photometric data used in this work are available only in electronic form at the CDS via anonymous ftp to cdsarc.u-strasbg.fr $(130.79 .128 .5)$ or via http://cdsweb.u-strasbg.fr/cgi-bin/qcat?J/A+A/466/743
Moutou et al. (2004, hereafter Paper I) using the FORS2 camera on the VLT.

OGLE-TR-132b occupies the edge of parameter space in several respects among the known transiting planets: it has a very short period and a rather large primary, within the uncertainties it is therefore probably the known gas giant receiving the highest flux from its host star, which also has a very high metallicity. The combination of these two factors, for instance, make OGLE-TR-132b a crucial object for the relation between star metallicity and planet core size proposed by Guillot et al. (2006). Therefore, refining the knowledge of its parameters is useful to provide constraints for hot Jupiter structure, formation and evaporation scenarios.

Given the faintness of OGLE-TR-132 $(I=15.7)$, large telescopes are necessary for the photometric and spectroscopic measurements.

In this paper, we present new high-resolution, high-S/N spectroscopic observations used to refine the parameters of the host star of OGLE-TR-132b, and high-precision photometric coverage of two transits, one year apart, to improve the accuracy of the planetary radius and the orbital ephemeris. The data were obtained with UVES on the VLT for the spectroscopy, and with 
FORS2 on the VLT (same data as in Paper I) and SUSI2 on the NTT for the photometry.

The methods to analyze the transit shape, estimate the uncertainties in the presence of photometric systematics and derive the stellar and planetary parameters are the same as in our previous studies of OGLE transiting planets (Bouchy et al. 2004, 2005; Pont et al. 2004, 2005, 2007; Moutou et al. 2004; Santos et al. 2006; Gillon et al. 2006) and will only be repeated briefly here when necessary. The reader is referred to those papers for further details.

\section{Observation and reduction}

\subsection{Spectroscopy}

High-resolution spectra of OGLE-TR-132 were obtained using the UVES spectrograph at the VLT-UT2 Kueyen telescope (program ID 076.C-0131). Eight exposures of $3000 \mathrm{~s}$ each were gathered between December 2005 and January 2006. Each individual spectrum was then combined using the $\mathrm{IRAF}^{1}$ scombine routine. The total $S / N$ obtained is close to 100 , as measured directly from small spectral windows with no clear spectral lines in the region near $6500 \AA$.

As in our previous spectroscopic study of other OGLE stars (Santos et al. 2006), the CCD was read in $2 \times 2$ bins for each exposure to reduce the readout noise and increase the number of counts in each bin. Similarly we opted for a slit width of 0.9 arcsec, which provides a spectral resolution $R=\lambda / \Delta \lambda \sim$ 50000. The observations were made using the Dichroic $390+580$ mode. The red portion of the spectra (used in this paper) covers the wavelength domain between 4780 and $6805 \AA$, with a gap between 5730 and $5835 \AA$.

As before, particular attention was paid to the orientation of the slit due to the relatively crowded field. The angle was chosen using the images available at the OGLE website ${ }^{2}$, so that no other star was present in the UVES slit during the observation.

The final spectrum of OGLE-TR-132 was used to derive stellar parameters and iron abundances. These were derived in LTE using the 2002 version of the code MOOG (Sneden 1973) ${ }^{3}$ and a grid of Kurucz Atlas plane-parallel model atmospheres (Kurucz 1993). The whole procedure is described in detail in Santos et al. (2006, and references therein) and is based on the analysis of $39 \mathrm{Fe}$ I and 12 Fe II weak lines and on imposing excitation and ionization equilibrium. The resulting parameters are listed in Table 1.

\subsection{Photometry}

\subsubsection{Observations}

The VLT/FORS2 data used here were presented in Paper I. NTT observations were obtained on April 19th, 2005 on the SUSI2 camera (programme 075.C-0462A). 272 exposures were acquired in a $5.4^{\prime} \times 5.4^{\prime}$ field of view. The exposure time was $60 \mathrm{~s}$, while the read-out time was $22 \mathrm{~s}$. The measured seeing varies between $0.75^{\prime \prime}$ and $1.38^{\prime \prime}$. The $R \# 813$ filter was used for all observations. We used SUSI 2 with a $2 \times 2$ pixel binning to get a good spatial and temporal sampling at the same time. The

\footnotetext{
${ }^{1}$ IRAF is distributed by National Optical Astronomy Observatories, operated by the Association of Universities for Research in Astronomy, Inc., under contract with the National Science Foundation, USA.

${ }^{2}$ http://www.astrouw.edu.pl/ ftp/ogle/index.html

${ }^{3}$ http://verdi.as.utexas.edu/moog.html
}
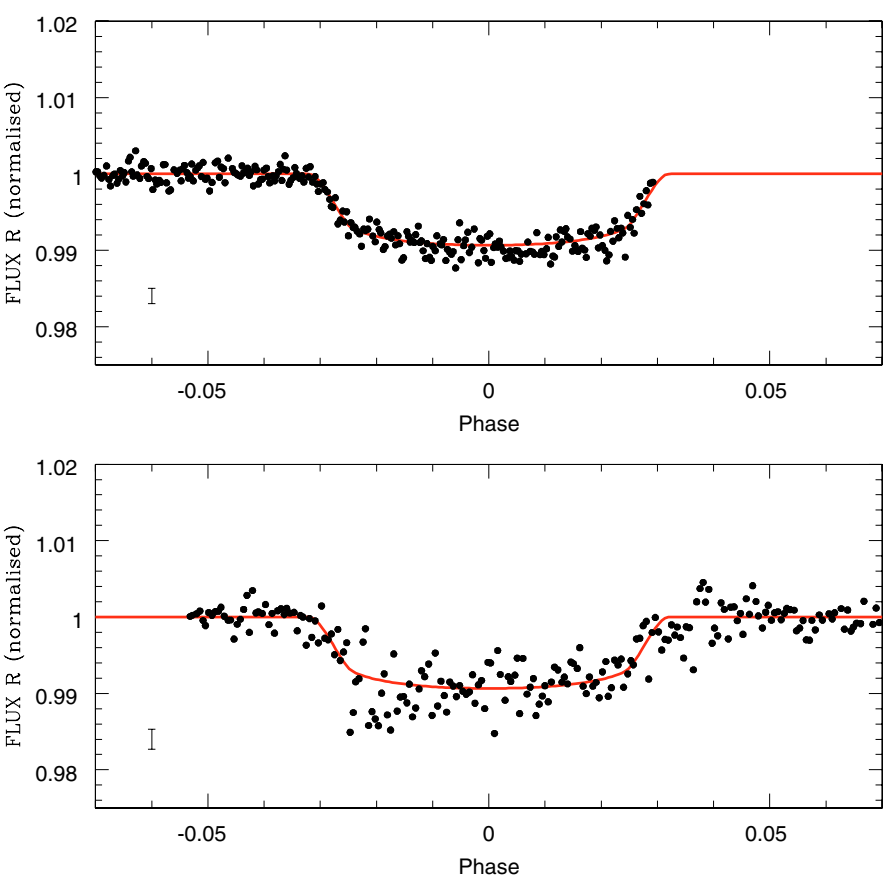

Fig. 1. VLT/FORS2 (top) and NTT/SUSI2 (bottom) light curves folded at the best phase and period. The best-fit transit curve is superimposed (solid line).

binned pixel size is $0.16^{\prime \prime}$. The airmass of the field decreases from 1.31 to 1.19 then grows to 1.54 during the sequence. The night was not photometric (large transparency variations).

The frames were debiassed and flatfielded with the standard ESO pipeline.

\subsubsection{Reduction}

Deconvolution-based photometry. We used the deconvolution-based photometric reduction method DECPHOT ${ }^{4}$, described in Gillon et al. (2006, 2007) and Magain et al. (2007) to analyze both VLT and NTT data. This method relies on the partial deconvolution of a set of images to the same higher resolution, leading to the minimisation of the systematic effects due to seeing variations along the run, the accurate determination of the sky background, and the detection of faint blending sources not visible in the original images. Furthermore, the Point-Spread Function (PSF) is determined simultaneously to the deconvolution of the images using all the sources present in the field. This PSF determination does not rely on the presence of any isolated star in the field, it is thus well suited for crowded field photometry.

Figure 1 presents the light curves obtained. The standard deviation of the VLT light curve before the transit is $1.12 \mathrm{mmag}$, while the mean photon noise is 1.04 mmag. Poor atmospheric conditions caused the NTT light curve to have worse precision than expected from the photon noise of the observations: the standard deviation of the NTT light curve after the transit is $1.60 \mathrm{mmag}$, while the mean photon noise is $1.30 \mathrm{mmag}$.

Image-subtraction photometry. In Paper I, the same VLT/ FORS2 data were reduced using a procedure based on the image subtraction ISIS software (Alard \& Lupton 1998; Alard $1999 ; 2000)$ and aperture photometry of the difference images.

\footnotetext{
${ }^{4}$ DEConvolution-based PHOTometry.
} 


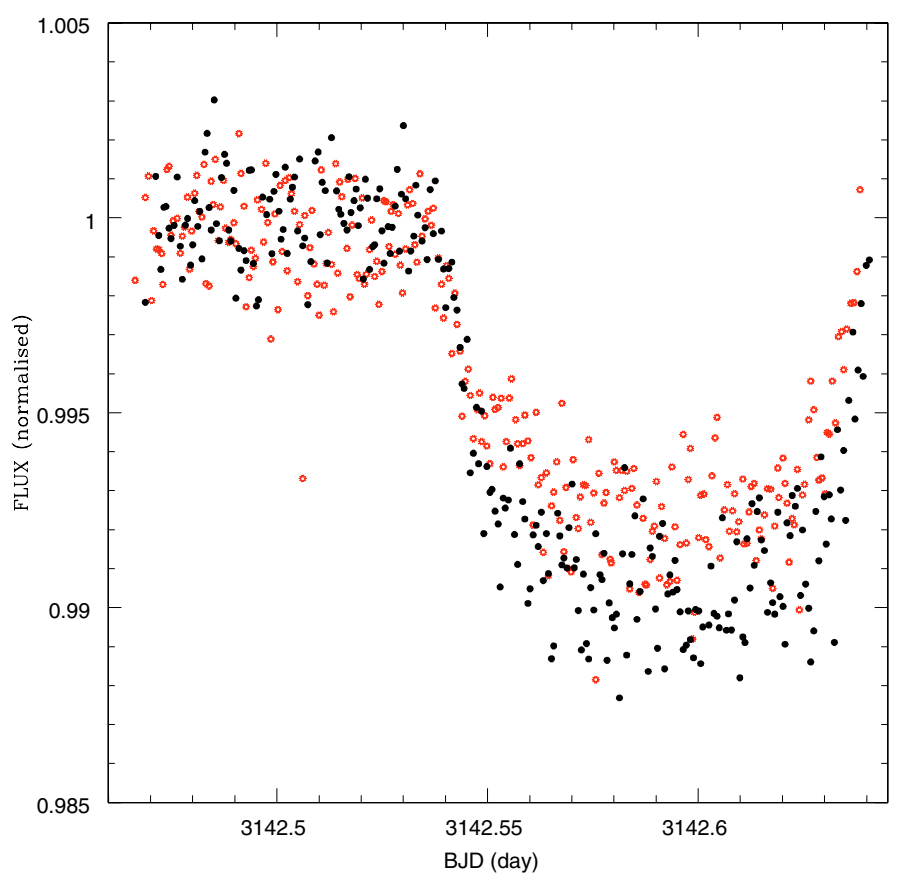

Fig. 2. The VLT/FORS2 light curve obtained in this analysis with DECPHOT (filled circles) compared to the one obtained in Paper I using an image subtraction procedure (open circles).

Comparing the light curve obtained with the one presented in Paper I, we find that both methods seem to give a similar precision, but a significantly different transit depth as can be seen in Fig. 2.

To investigate this discrepancy, we first decided to check the reduction procedure used in Paper I. As explained in Alard \& Lupton (1998), ISIS relies on the use of a high-S/N reference image which is convolved to the seeing of the analyzed image and subtracted, leading to a difference image. The flux in this image is then measured by aperture or profile photometry. This kind of reduction method is generally presented as very suitable for crowded field photometry, as variability is measured on a nearly empty surface, while other reduction methods aim at measuring the total flux of objects which could be strongly blended (Udalski et al. 2002). In Paper I, the flux in the reference image and in the difference images were measured with aperture photometry, using the same aperture (10 pixels) for all images of the run.

It appears that with this procedure, a tradeoff has to be made between the relative precision of the light curve and the reliability of the amplitude of the flux variation, as illustrated in Fig. 3. This figure shows light curves obtained with the procedure used in Paper I and different apertures. It clearly shows that using smaller apertures leads to an artificial reduction of the amplitude of any variation in the light curve, and thus also of the standard deviation, an effect already reported by Hartman et al. (2004). This normalisation problem explains the observed discrepancy. The best light curve in Fig. 3 having a transit depth in agreement with the one obtained with DECPHOT (aperture $=14$ pixels) has a standard deviation outside the transit $\sim 1.7 \mathrm{mmag}$. This curve and the one presented in Paper I are compared to the DECPHOT curve in Fig. 4.

With image subtraction methods, one uses the stellar flux measured in the reference image to convert the differential flux units into magnitudes. Any difference between the fraction of the stellar flux measured in the difference images and in the
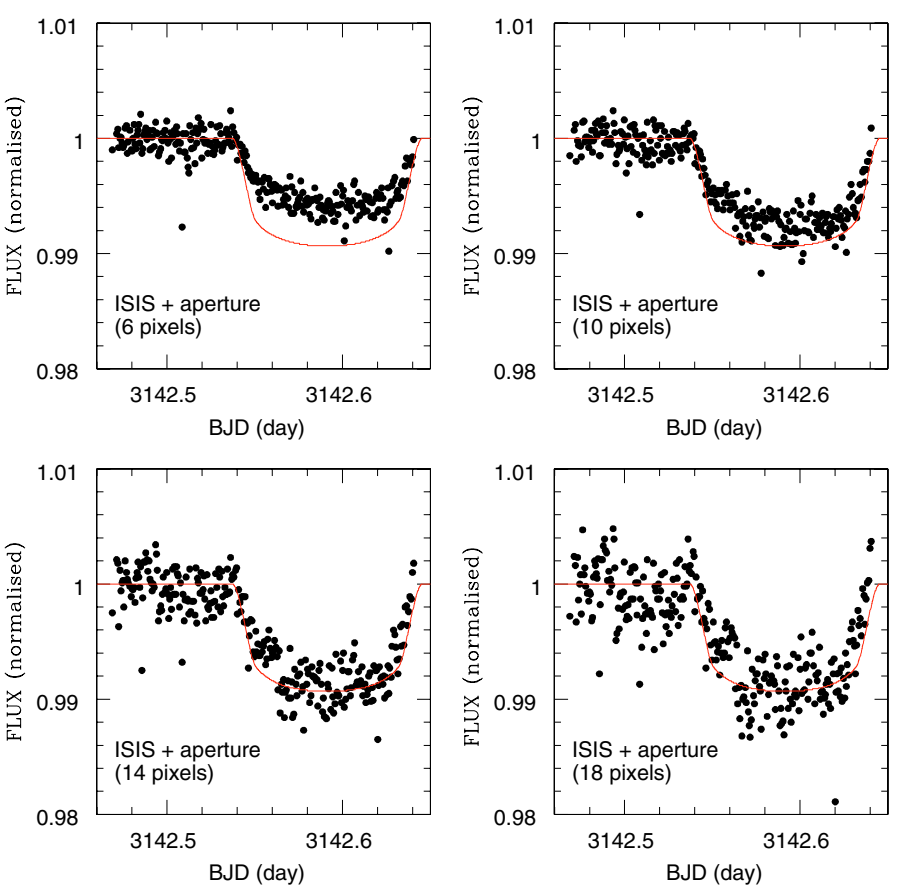

Fig. 3. VLT/FORS2 light curves for OGLE-TR-132b and obtained with the same reduction procedure as in Paper I, using 4 different apertures. The best-fit transit curve obtained after reduction with DECPHOT is superimposed (solid line).

reference image is thus able to artificially modify the scale of the light curve. This was the case for the reduction procedure used in Paper I: the fraction of the stellar flux within the aperture was lower in the average difference image than in the reference image, as the latter is the best-seeing image of the run. This normalistion problem can be avoided by using PSF fitting to measure the flux within difference and reference images. A PSF fitting algorithm called phot. csh and designed to measure the difference flux is implemented in the ISIS pipeline, but it was not used in Paper I.

One of us (J.D.H.) reduced the VLT/FORS2 data with his own ISIS-based reduction method (see Hartman et al. 2004 for a thorough description). In this method DAOPHOT is used to obtain the fluxes on the reference image via PSF fitting, an aperture correction is then performed to transform the PSF magnitudes to aperture magnitudes measured through a fixed aperture size. That same aperture size is used for the PSF normalization radius (rad_aper) in phot.csh to ensure that the flux scale is the same for the reference and subtracted images. The resulting light curve is also shown in Fig. 4. The transit depth obtained is in good agreement with the one obtained with DECPHOT. The standard deviation outside the transit is $\sim 1.5 \mathrm{mmag}$, still significantly higher than the one from deconvolution-based photometry. Furthermore, the filtering done to remove points that came from images with poor subtractions left only 224 points out of a total of 281 , i.e. $20 \%$ of the data were rejected by the procedure.

Aperture photometry. Figure 5 shows that no close companion contaminates the PS F core of OGLE-TR-132 in the deconvolved best-seeing VLT image. This relative isolation allowed us to reduce the VLT data with aperture photometry and to check the agreement between the resulting light curve and the one obtained with DECPHOT. For this purpose, we used the DAOPHOT aperture photometry software (Stetson 1987) with 


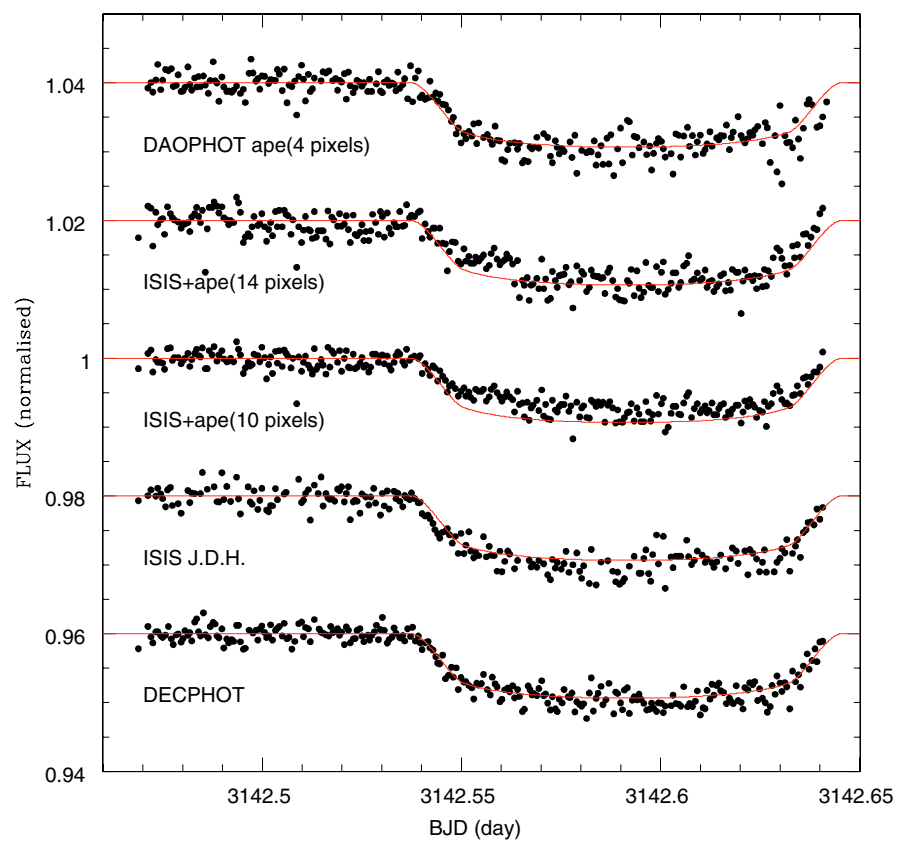

Fig. 4. The VLT/FORS2 light curves obtained in this analysis with, from top to bottom, DAOPHOT aperture photometry, ISIS + aperture photometry using 2 different apertures, obtained by J.D.H. using his own ISIS-based procedure, and with DECPHOT. The curves are shifted vertically for clarity. The best-fit transit curve obtained after reduction with DECPHOT is superimposed (solid line). See text for further details.

a rather small aperture (4 pixels) to avoid any dilution of the transit signal and noise increase due to the faint blending companion just East of the target star. Figure 5 compares the best light curve obtained with the ones from the other methods. The agreement with the DECPHOT light curve is excellent, while the precision obtained is similar to the one obtained by J.D.H. with his ISIS-based method (standard deviation $\sim 1.5 \mathrm{mmag}$ ).

\section{Results}

\subsection{Transit parameters}

We fit the joint VLT and NTT light curves using the Mandel \& Agol (2002) transit model with quadratic limb-darkening coefficients from Claret (2000). The results are given in Table 1. As in Bouchy et al. (2005), we use the radius ratio, the impact parameter (minimum projected distance from the center of the planet to the center of the star divided by the star's radius), and $V_{\mathrm{T}} / R$ (the transverse velocity of the planet at the center of the transit in terms of the star's radius). These three parameters are independent of any assumption for the size and mass of the primary. We have used the circular residual shift method explained in Moutou et al. (2004) to estimate error bars on the parameters that take into account possible trends in the photometry. We checked that the differences between the values fitted independently on each transit were compatible within the uncertainty estimates of the combined fit, which suggests that the uncertainties are realistic.

If the sum of the two limb-darkening coefficients is left as a free parameter, it converges to a value that is 0.1 lower than the coefficients in Claret (2000), with an uncertainty of similar amplitude. In our adopted solution, we fix the limb-darkening coefficients to their tabulated values.
Table 1. Parameters for the OGLE-TR-132 system, host star and transiting planet.The uncertainty on the stellar mass is given in bracket to emphasis the fact that it does not include the uncertainties on stellar evolution models that could increase the error bar.

\begin{tabular}{ll}
\hline \hline Star & \\
$T_{\text {eff }}[\mathrm{K}]$ & $6210 \pm 59$ \\
$\log g[\mathrm{cgs}]$ & $4.51 \pm 0.27$ \\
{$[\mathrm{Fe} / \mathrm{H}]$} & $0.37 \pm 0.07$ \\
& \\
Mass $\left[M_{\odot}\right]$ & $1.26 \pm(0.03)$ \\
Radius $\left[R_{\odot}\right]$ & $1.34 \pm 0.08$ \\
& \\
Transit & \\
& \\
Impact parameter & $0.53 \pm 0.09$ \\
Radius ratio & $0.0916 \pm 0.0014$ \\
$V_{\mathrm{T}} / R$ & $17.79 \pm 1.15$ \\
& \\
Planet & \\
& \\
Mass $\left[M_{\mathrm{J}}\right]$ & $1.14 \pm 0.12$ \\
Radius $\left[R_{\mathrm{J}}\right]$ & $1.18 \pm 0.07$ \\
Density $\left[\mathrm{g} \mathrm{cm}{ }^{-3}\right]$ & $0.86_{-0.21}^{+0.28}$ \\
Period $[$ days $]$ & $1.689868 \pm 0.000003$ \\
$T_{\text {tr }}[\mathrm{BJD}]$ & $2453142.5912 \pm 0.0003$ \\
\hline
\end{tabular}

\subsection{Host star parameters}

To estimate the mass and radius of the host star from its spectroscopic parameters, some additional assumptions are needed. As in previous papers (see Introduction), we assume that the star follows the stellar evolution models of Girardi et al. (2002) and we combine the constraints from the spectroscopic and transit parameters by Maximum Likelihood. We point out that the derived mass and radius of the star, and therefore of the planet, will be correct only to the extent that these models are accurate. For such a high metallicity, significant discrepancies are not excluded. However, our high-precision photometric data helps lifting the degeneracy between the star's radius and the impact parameter, providing a constraint on the star's radius that is independent of the models.

The 1-sigma interval for the stellar evolution models from the Maximum Likelihood solution is $0-2$ Gyr. The upper limit is slightly higher than that estimated by Moutou et al. (2004), $1.5 \mathrm{Gyr}$, because of the lower temperature we obtain for the primary. From a measurement of the calcium emission line for this star from the same UVES spectra, Melo et al. (2006) estimate that it must be older than 0.5 Gyr. Therefore, we estimate that OGLE-TR-132 has an age in the 0.5-2 Gyr range.

\subsection{Planetary mass and radius}

The planetary radius is obtained from the spectroscopically determined stellar radius and the photometrically determined radius ratio. The planetary mass is obtained from the stellar mass and the radial velocity semi-amplitude. Although we had no new radial velocity data compared to Bouchy et al. (2004), our data also lead to a slight change in the estimated mass because of the improved ephemeris.

Table 1 gives the derived values for the stellar and planetary mass and radius, and the planet density. 


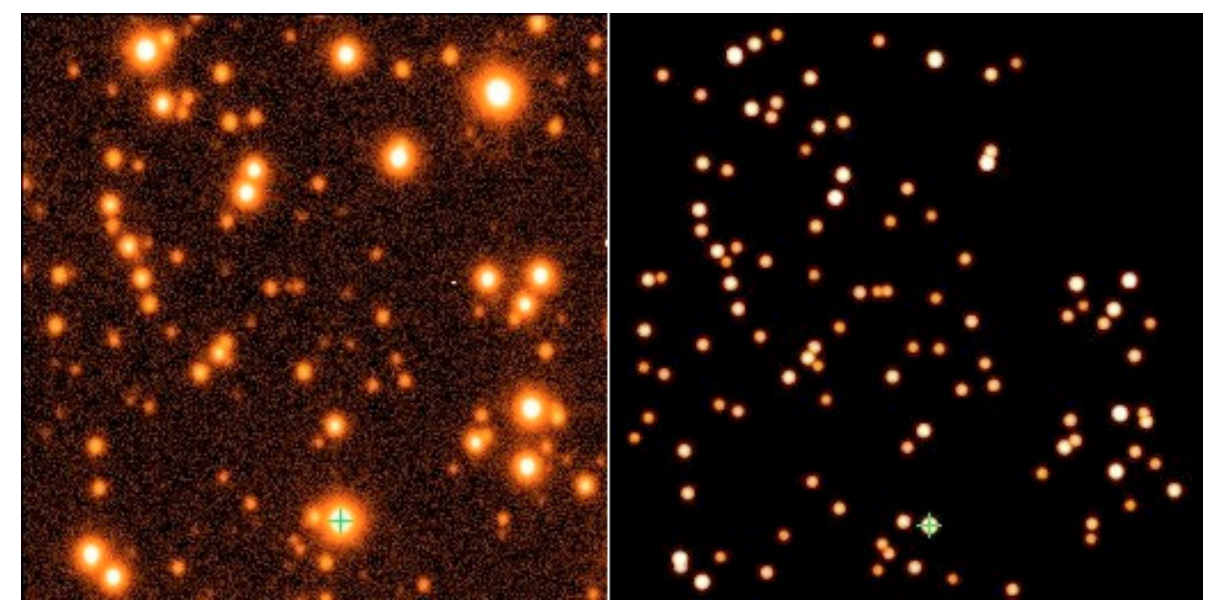

Fig. 5. Left: OGLE-TR-132 (marked with a cross) in a 256 pixels $\times 256$ pixels sub-image $\left(0.51^{\prime} \times 0.51^{\prime}\right)$ from the best seeing VLT/FORS2 image of the run (top $=$ North, left $=$ East). Right: deconvolved image.

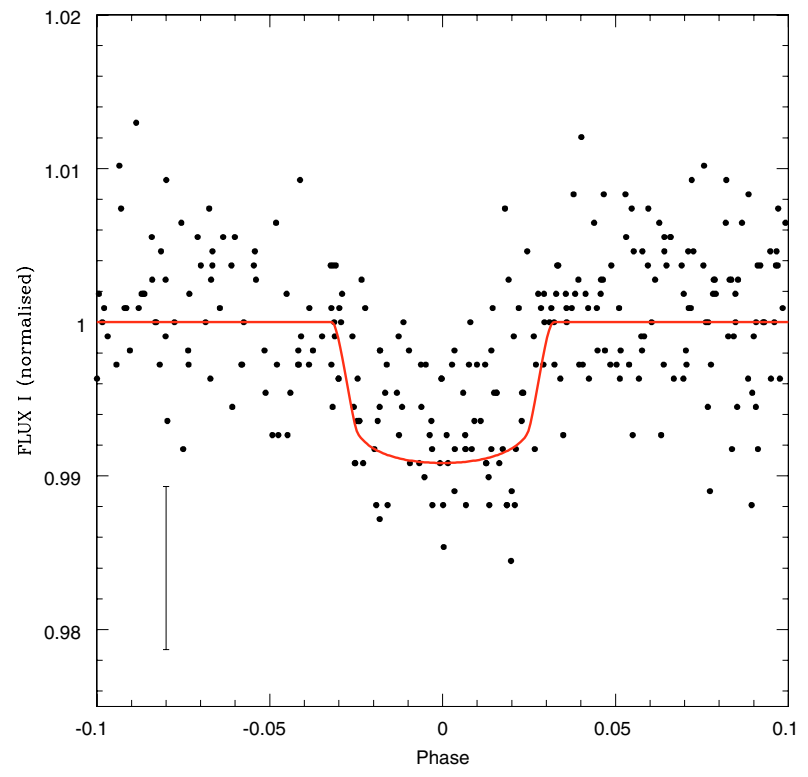

Fig. 6. OGLE data folded at the best phase and period from the VLT and NTT light curves, with the best-fit transit curve superimposed (solid line).

\subsection{Ephemeris}

The uncertainty on the central epoch is about $25 \mathrm{~s}$ for the VLT transit and $50 \mathrm{~s}$ for the NTT transit. If the transits are strictly periodic, this translates into an uncertainty of $3 \times 10^{-6}$ days $(0.26 \mathrm{~s})$ on the period.

By fitting the transit shape obtained from Table 1 on the original OGLE data, with only the epoch and period as free parameters, we obtain $P=1.68985 \pm 0.00001$ days from the OGLE photometry (uncertainty assuming white noise only). This is in agreement with the difference between the epochs of the VLT and NTT transits, and shows that any time delay effect between the two does not exceed an amplitude of $\sim 6$ min. Using Eq. (33) from Agol et al. (2005) and assuming that the phase difference between both transits leads to a maximal time delay, this corresponds to a $4 M_{\oplus}$ exterior planet in 2:1 mean-motion resonance. A lighter planet in this configuration cannot be excluded, nor can a heavier one in another orbital and/or phase configuration.

Figure 6 shows the OGLE data folded at the best phase and period from the VLT and NTT light curves.

\section{Discussion}

\subsection{Photometry}

The transit depth is found to be deeper than indicated in Paper I (radius ratio $=0.0916 \pm 0.0014$ vs. $0.0812 \pm 0.0017$ in Paper I). As detailed in Sect. 2.2, this is due to a normalisation problem with the photometry procedure used in Paper I, leading to an artificially low rms and transit depth. This problem does not seem to be inherent in image subtraction photometry, in the sense that it can be avoided by using an improved reduction procedure, as shown by the reduction done in this work with the ISIS software.

The precision obtained here with image subtraction is significantly worse than that obtained with DECPHOT. This can be explained by several drawbacks of the image subtraction method which are not present in DECPHOT:

- The first one is the fact that the matching of a reference image and an analyzed image could only give a correct connecting kernel and background correction if the photometry of each light source is obtained separately from the others. This is not the case with this method, since only a simple flux scaling factor is determined which is correct globally, but is wrong to a significant extent locally. Indeed, the flux scale factor between both images is actually not the same for every stars. The global agreement obtained when the merit function is minimized can thus be far from the real solution: the inaccuracy of the background correction, the kernel and the unphysical scaling factor can compensate each other, leading not only to a lower photometry precision, but also to the presence of correlated noises in the photometry and to possible normalisation problems. The only way to achieve a local and realistic agreement between the modified master image and the analyzed image should be to iterate on the photometry and the astrometry of each star.

- The kernel tries to connect two images of the same run which can be very close in resolution: the PSF width difference between both can be much lower than the sampling interval (the pixel). Thus, the sampling of this connecting kernel, and thus its accuracy, can be very poor, even if many stars are used to determine it. Furthermore, if we consider a PSF which has a spatial width in one dimension lower than the one of the PSF of the master image, there is no mathematical solution to the problem: no convolution kernel is able to connect one to the other. In terms of kernel accuracy, it is much better 
to connect a much higher resolution image to the analyzed image.

- The kernel is modelised as a simple analytical function, while it can far to be analytical in real cases.

- Any difference in the fraction of flux measured in the difference images and the reference image can lead to a normalisation problem, i.e. to a decrease or to an increase of the photometric variations and thus of the transit depth and of the standard deviation. This can happen relatively easily, as difference images contain weak unblended point sources and the reference image contain more blended and bright sources.

The precision obtained here with image subtraction is comparable to that obtained with simple aperture photometry. Nevertheless, the analyzed star is relatively isolated, and we can expect that better precisions would be obtained with image subtraction photometry than with aperture photometry for a more blended target. Deconvolution-based photometry is superior to both methods in this case. As the principle of the method makes it particulary suitable for crowded field photometry and that very precise results have already been obtained for more blended stars (Gillon et al. 2006, Pont et al. 2007), we expect that DECPHOT should keep this advantage for more crowded fields. Therefore, it may be beneficial to use DECPHOT for performing the photometric reduction of transit surveys. Indeed, the method should not only be able to decrease significantly the deviation within the light curves, but also to reduce drastically the topological red noise, i.e. the red noise coming from the environment of the analyzed star. As shown by Pont, Zucker \& Queloz (2006), the red noise level is the main limitation of the existing ground-based surveys. Nevertheless, in its present form, DECPHOT is $\sim 10$ to 100 times slower than ISIS and $\sim 100$ to 1000 times slower than aperture photometry, and it also requires much more user intervention. Its application to transit surveys thus relies on the improvement of its speed and ease of use, a task which is now in progress.

\subsection{The OGLE-TR-132 system}

The present work provides a very precise determination of the shape of the planetary transit light curve of OGLE-TR-132, and highly precise ephemeris that will allow to predict precise transit timings for many years. The spectroscopic parameters of the host star are also significantly improved.

Compared to previous estimates, the new data indicate that OGLE-TR-132 is a slightly cooler star, confirmed as very metal-rich. The effect of a cooler star, leading to a smaller estimated primary radius, and of a deeper transit, leading to a larger radius ratio, compensate to first order, so that our value of the planetary radius is only slightly larger than that reported in Paper I, and compatible within the error bars $\left(1.18 \pm 0.07 R_{\mathrm{J}}\right.$ vs. $1.13 \pm 0.08 R_{\mathrm{J}}$ in Paper I). The cooler temperature also implies a decrease in the estimated stellar mass, with a corresponding decrease in the planetary mass $\left(1.14 \pm 0.12 M_{\mathrm{J}}\right.$ vs. $1.19 \pm 0.13 M_{\mathrm{J}}$ in Paper I), also within the error bars (the uncertainty on the planetary mass is dominated by that of the amplitude of the radial velocity orbit).

The baseline provided by our light curves for the detection of transit timing delay effects, due to the presence of a second planet or a satellite, is of the order of a minute. This is less constraining than in the similar case of OGLE-TR-113 (Gillon et al. 2006) because here the photometric precision is similar but the transit is much shallower. In this case, a several Earth-mass satellite would not be detectable even in a favourable configuration (obviously, any satellite is also very unlikely given the very small orbital distance).

Acknowledgements. The authors thank the ESO staff on the VLT and NTT telescopes for their diligent and competent execution of the observations. Support from the Fundação para a Ciência e a Tecnologia (Portugal) to N.C.S. in the form of a fellowship (reference SFRH/BPD/8116/2002) and a grant (reference POCI/CTE-AST/56453/2004) is gratefully acknowledged.

\section{References}

Agol, E., Steffen, J., Sari, R., \& Clarkson, W. 2005, MNRAS, 359, 567

Alard, C. 1999, A\&A, 343, 10

Alard, C. 2000, A\&AS, 144, 363

Alard, C., \& Lupton, R. H. 1998, ApJ, 503, 325

Bouchy, F., Pont, F., Santos, N. C., et al. 2004, A\&A, 421, L13

Bouchy, F., Pont, F., Melo, C., et al. 2005, A\&A, 431, 1105

Claret, A. 2000, A\&A, 363, 1081

Gillon, M., Pont, F., Moutou, C., et al. 2006, A\&A, 459, 249

Gillon, M., Magain, P., Chantry, V., et al. 2007, in Transiting Extrasolar Planets Workshop, ed. C. Afonso, D. Weldrake, \& T. Henning

Girardi, M., Manzato, P., Mezzetti, M., et al. 2002, ApJ, 569, 720

Guillot, T., Santos, N. C., Pont, F., et al. 2006, A\&A, 453, L21

Hartman, J. D., Bakos, G., Stanek, K. Z., \& Noyes, R. W. 2004, ApJ, 128, 1761

Kurucz, R. 1993, ATLAS9 Stellar Atmosphere Programs and $2 \mathrm{~km} \mathrm{~s}^{-1}$ grid, Kurucz CD-ROM No. 13 (Cambridge, Mass.: Smithsonian Astrophysical Observatory)

Magain, P., Courbin, F., Gillon, M., et al. 2007, A\&A, 461, 373

Mandel, K., \& Agol, E. 2002, ApJ, 580, 171

Melo, C., Santos, N. C., Pont, F., et al. 2006, A\&A, 460, 251

Moutou, C., Pont, F., Bouchy, F., \& Mayor, M. 2004, A\&A, 424, L31

Moutou, C., \& Pont, F. 2006, Formation planétaire et exoplanètes, Ecole thématique du CNRS, Goutelas (Loire), 23-27 mai 2005, ed. J.-L. Halbwachs, D. Egret, \& J.-M. Hameury (Strasbourg: Observatoire astronomique de Strasbourg et Société Française d'Astronomie et d'Astrophysique (SF2A)), 55

Pont, F., Bouchy, F., Queloz, D., et al. 2004, A\&A, 426, L15

Pont, F., Bouchy, F., Melo, C., et al. 2005, A\&A, 438, 1123

Pont, F., Zucker, S. , \& Queloz, D. 2006, MNRAS, 373, 231

Pont, F., Moutou, C., Gillon, M., et al. 2007, A\&A, 465, 1069

Santos, N. C., Pont, F., Melo, C., et al. 2006, A\&A, 450, 825

Sneden, C. 1973, Ph.D. Thesis, Univ. of Texas

Stetson, P. B. 1987, PASP, 99, 111

Udalski, A., Paczyński, B., Zebrun, K., et al. 2002, Acta Astron., 52, 1

Udalski, A., Pietrzynski, G., Szymanski, M., et al. 2003, Acta Astron., 53, 133 